\title{
Más allá del formato. Una mediateca virtual como propuesta de emprendimiento cultural para creadores emergentes ${ }^{*}$
}

\author{
Bibiana Paola Ríos Cortés ** \\ Recibido: 2014-10-30 - Aprobado por pares: 2015-01-03 \\ Enviado a pares: 2014-11-26 - Aceptado: 2015-02-05
}

\section{Resumen}

Este artículo explora aproximaciones teóricas al concepto de cultura, su relación con la globalización, y sus implicaciones en el desarrollo del emprendimiento cultural en países como Colombia. Desde el emprendimiento cultural, se consideran diferentes postulados teóricos, hasta posibles aplicaciones a partir del uso de las tecnologías de la información y la comunicación. Una mediateca virtual es una de esas potenciales aplicaciones, y son los productos de los estudiantes de áreas como la comunicación, el diseño, la publicidad y el mercadeo, el insumo para la construcción de un repositorio digital, que en una segunda fase será la propuesta de una plataforma para la visibilidad, la divulgación y el emprendimiento cultural de creadores emergentes, en un interés de generar estrategias para la construcción de la memoria en torno a la comunicación.

Palabras clave: emprendimiento cultural, mediateca, globalización, capitalismo mediológico

Este artículo se origina en el proyecto de investigación "Mediateca de la comunicación", de la Facultad de Comunicación de la Fundación Universitaria Unipanamericana, encaminado a crear un espacio virtual para almacenar y publicar los ejercicios académicos realizados en dicha Institución.

** Bibiana Paola Ríos Cortés. Maestrante en Gestión de la Cultura y Mercadeo del Arte. Especialista en Gerencia de Diseño. Diseñadora Visual. Docente investigadora-Grupo de investigación en Comunicación, Medios y Mercadeo. Correo Electrónico: bpriosc@unipanamericana.edu.co." 


\title{
Beyond the Format. \\ A Virtual Multimedia Library as a Cultural Entrepreneurship Proposal for Emerging Creators
}

\begin{abstract}
This article explores the theoretical approaches to the culture as concept, its links with globalization, and the cultural entrepreneurship development in countries like Colombia. From the cultural entrepreneurship perspective, the definitions related to theoretical propositions to the potential applications based on the use of media and communication technologies are considered. A virtual multimedia library is the outcome of these relationships; the products in the design, advertising and marketing areas by students is the main supply to build a digital repository, a platform for exposition, spreading and cultural entrepreneurship for emerging artists in the near future that also make memory around communication possible to be constructed.
\end{abstract}

Key words: Cultural entrepreneurship, Multimedia library, Globalization, Capitalism mediological. 


\section{INTRODUCCIÓN}

Según la Declaración Universal de la Diversidad Cultural (Unesco, 2001), la cultura puede definirse como el conjunto de rasgos distintivos espirituales y materiales, intelectuales y afectivos que caracterizan a una sociedad y que pueden abarcar, además, los modos de vida, los valores, las tradiciones y las creencias de un grupo social. La cultura, al ser el escalón más alto del patrimonio de una sociedad, debe ir progresivamente adaptándose también a las necesidades de esas mismas sociedades y procurar por que el diálogo con el desarrollo, esté cada vez más renovado.

En esa renovación, la cultura está peligrosamente relacionada con la economía del conocimiento. La Unesco, por ejemplo, en el informe sobre las políticas para la creatividad, cuenta las preocupaciones relacionadas con el enriquecimiento cultural, a partir de la globalización, donde el desequilibrio entre los imperativos de la cultura y los intereses de la economía provoca una constante de incertidumbres sobre el devenir cultural y sus posibles aplicaciones desde el emprendimiento (Unesco, 2010).

La producción de bienes y servicios cuyo origen está en la creación y la cultura se sitúa entre las principales fuentes de innovación e intercambio en el contexto global. Ahora mismo, no se discute sobre si es viable o no el emprendimiento cultural; más bien, se debate cuál es la mejor manera, de hacer uso de los recursos sin que la economía esté por encima del bien cultural. Esta nueva fase económica es lo que Mattelart denomina capitalismo mediológico, en donde la unificación de la información y del dinero da lugar a nuevas relaciones sociales y humanas, centrándose en el mundialismo comunicativo (Mattelart, 1998).

En el mundialismo comunicativo, las distribuciones específicas de poder, la dominación y la subordinación de las sociedades actuales transforman la cultura en un fenómeno que puede ser hegemonizado. La realidad de cualquier supremacía es que siempre es dominante, pero nunca de un modo absoluto (Williams, 1980). Las dominaciones se presentan desde un entrecruzamiento de fuerzas, de las que el dominado casi nunca es consciente. Las formas de expresión, los sistemas de significación y los mecanismos a través de los cuales la clase dominada sobrevive son los principios en los que el marxismo encuentra su lugar.

Referirse al concepto de cultura dentro de la globalización es hablar de políticas claras de recuperación y conservación, donde la responsabilidad de asegurar la herencia cultural a las generaciones presentes y futuras está cada vez más amenazada. Esta herencia cultural comprende todos los bienes que son expresiones y testimonios de la creación humana. Museos, obras de arte, lugares, restos arqueológicos, colecciones zoológicas, botánicas o geológicas, libros, manuscritos, documentos, partituras y discos, fotografías, producción cinematográfica y objetos culturales en general cuentan la evolución de la vida social y económica de cada nación y sus individuos.

Esta visión pragmática invita a reflexionar sobre las nuevas prácticas de la cultura, pone su mirada en la historia y en la sociedad, e intenta generar una postura crítica, 
dando respuestas creativas a las problemáticas actuales. Se alinea con todas aquellas colectividades, que más que atención necesitan un espacio activo y contingente, que les permita reconocerse como parte de un grupo, de una nación o de un continente.

Estas nuevas prácticas de la cultura incluyen la globalización como un aspecto y fenómeno mucho más amplio, que afecta a todas las dimensiones de la condición humana y está ligada a la generalización de valores culturales. Con la globalización, se da inició a las nuevas configuraciones de región en los ámbitos político, económico, social y cultural, que establecerán nuevos procesos estructurales en la mayoría de los países llevándolos a la conformación de bloques económicos, como plataformas de desarrollo. La globalización es un hecho concreto que ha redefinido las relaciones internacionales y ha creado nuevos y poderosos patrones culturales (Castells, 2005).

En respuesta a la globalización, las industrias culturales son estrategias que dan un valor agregado tanto en el nivel económico como en el social a las naciones y sus individuos, y constituyen una forma de conocimiento que se traduce en empleos y mejores estándares de vida. El mayor riesgo en la actualidad no es la hegemonía cultural; son las diferencias que se han construido entre la manera en la que se compra y la que se vende el bien cultural; allí entran en juego la concentración de los mercados y el diálogo con los creadores de los bienes.

Proteger la producción cultural de las naciones, frente a promover el emprendimiento cultural es el camino al que muchas de las industrias culturales le apuestan en la actualidad. La Unesco, en sus políticas para la creatividad, considera que la respuesta de los países emergentes y de economías intermedias a la promoción de la diversidad exige una actitud proactiva, capaz de convertir la dinámica generada por la globalización, en una herramienta participativa y de desarrollo productivo que evidencie la inyección de los valores de cada cultura local, en el torrente de lo global (Unesco, 2010). Si la globalización se ve como la inyección de cada cultura en la perspectiva de lo global, sin que cada parte pierda su esencia y su identidad, entonces, estamos hablando de una globalización, cuya prioridad es lo particular.

Los museos se encuentran entre las instituciones culturales más importantes, debido a la gran cantidad de objetos, con alto grado de valor cultural e histórico que albergan. Los museos, transformados en lo que hoy conocemos, gracias al uso de la tecnología, se convierten en bibliotecas, centros de documentación, repositorios de imágenes fijas y audiovisuales; además, son puntos de análisis estratégico por parte de instituciones académicas, empresas, expertos y profesionales, para investigar tanto sobre el pasado, como sobre el presente y el futuro de la memoria (IMPIVA Disseny, 2009).

Cuando se define al museo desde la concepción tradicional del espacio físico, es necesario remontarse al primer mouseion, fundado alrededor del 290 a. C. en Alejandría por Tolomeo I Sóter. Este mouseion era un gran edificio con salas de lectura, bibliotecas y observatorios, donde se reunían los sabios y eruditos a reflexionar sobre el futuro de la humanidad. 
Hoy la concepción de museo sobrepasa el formato y facilita la interacción directa con las obras y sus creadores; genera nuevas formas de comunicación, y dinamiza los lenguajes desde los que se exploran los conceptos de entornos digitales y centros de memoria.

El museo como centro de documentación y memoria, combinado con las formas en las que el usuario se conecta con las obras y sus creadores, se denominará para este artículo mediateca virtual, pues, aunque persigue la construcción de la memoria, la mediateca no guarda la información de manera estática; por el contrario, permite que el usuario recorra el espacio e interactúe con el mismo, como si estuviera recorriendo un museo físico.

En una mediateca virtual, se exploran las nuevas relaciones entre el arte y la cultura; se habla de nuevos lenguajes, donde se reivindican los encuentros y desencuentros entre el artista y el receptor. Estas nuevas dinámicas de las mediatecas cuentan cómo la visita a una exposición no tiene rutas lineales, y al mismo tiempo cómo las obras no contienen significados fijos. Las mediatecas, deben mostrar las obras más allá del formato.

¿Pero cómo puede darse el emprendimiento cultural? ¿Cómo puede la cultura ser eje de desarrollo sostenible en una sociedad? ¿Cómo se da el proceso de transformar actividades culturales en activos económicos? La respuesta quizá no esté aquí, ni en ningún célebre documento que englobe todos los grandes aportes de la cultura a una sociedad. La respuesta debe darse desde las formas en las que el emprendimiento cultural se hace tangible, y en este sentido, ¿puede una mediateca virtual, hacer parte de las nuevas configuraciones del emprendimiento cultural?

La mediateca deja lo solemne de la biblioteca tradicional, para transformarse en un centro de información navegante. Las nuevas prácticas culturales, en las que se inscribe la mediateca, están asociadas con las nuevas tecnologías que modifican el contexto de lo contemporáneo. Estar al día de las exigencias de esta clara mezcla entre lo tecnológico y lo cultural exige una postura crítica que permite hacer buen uso de ambas, sin perder lo fundamental de la identidad.

Las instituciones académicas, y vale la pena aclarar, las instituciones de Educación Superior, a través de la gestión investigativa, deben generar acciones puntuales para que los proyectos de sus estudiantes y docentes no mueran en los anaqueles de las bibliotecas; estos proyectos deben trascender de las fronteras del soporte para ser visibles, y dar solución a problemas reales. Una mediateca en el área de la comunicación, por ejemplo, puede ser la respuesta a muchos interrogantes sobre el devenir de las áreas afines como la publicidad, el mercadeo, el diseño, el periodismo, el arte; un futuro que se asocie al reconocimiento de los actores, de las formas en cómo se ejerce cada disciplina; pero ante todo, el reconocimiento de lo particular de cada profesión, contribuyendo de manera importante a la protección de la producción cultural del país (Bonilla, Cabrera, Crespo, Cristiano, Ríos \& Sánchez, 2012).

¿Puede una mediateca virtual, hacer parte de las nuevas configuraciones del emprendimiento cultural para creadores emergentes? La respuesta a esta pregunta debe darse a partir de un eje temático desde el cual se desarrollará este artículo y es lo que 
determinará, el diseño y desarrollo de una plataforma cultural para que creadores emergentes, puedan emprender y contribuir a la construcción y consolidación de la memoria en torno a la comunicación.

\section{METODOLOGÍA}

En el artículo "Más allá del formato, una propuesta de mediateca para creadores emergentes" se estructura una metodología de tipo mixto, que parte de un análisis exploratorio, constituido por dos etapas; en la primera, en este caso de tipo cualitativo, se genera una serie de hallazgos, que contribuyen en el desarrollo de la segunda etapa cuantitativa. El supuesto en el cual se basa el uso de este diseño es que la exploración es un requisito necesario para la segunda fase del estudio. Desde el enfoque cualitativo se tienen en cuenta: la definición del problema, el diseño de la metodología, la recolección de la información, el análisis de la información y finalmente la validación de la misma. Durante el proceso, se plantea un diálogo constante con el objeto de estudio, que conecta la postura de diferentes autores en relación con la cultura, el emprendimiento, la gestión cultural, las industrias culturales y los museos, además del uso de las nuevas tecnologías en la apropiación del conocimiento. Esta información se obtiene del análisis simultáneo de bases de datos, relacionadas con el área de la comunicación y el diseño. El aporte de expertos en las áreas de museología y tecnologías de la información, complementa el panorama teórico que soporta la investigación.

En el análisis de variables cuantitativas, se comparan los resultados como producto de las encuestas realizadas a los estudiantes y relacionadas con el desarrollo de la mediateca en la Fundación Universitaria Panamericana. Al final, las variables cualitativas y cuantitativas se cruzan de tal manera que permitan analizar el objeto de estudio y generen una respuesta coherente a los objetivos planteados al inicio de la investigación.

\section{RESULTADOS}

\section{APROXIMACIÓN AL CONCEPTO DE CULTURA}

Se definirá la cultura como punto de partida, en el análisis de las variables para el estudio de las industrias culturales.

El término cultura aparece para plantear una oposición entre la creación humana y la naturaleza; es decir, intenta dar una explicación al modo de vivir de los seres humanos y sus comportamientos. Calicles, en el diálogo platónico de Georgias, concibe la cultura, como la transición histórica entre lo rural y lo urbano, y se acerca a la primera definición del término. Esta concepción, aunque profundamente crítica, está directamente relacionada con el territorio y aísla al individuo, como ser particular, de las diferentes manifestaciones que se concretan cuando se hace parte de un grupo social.

La antropología social, por otra parte, habla de la cultura como el resultado de todo lo producido por el hombre, y estudia las sociedades humanas contemporáneas y sus 
formas de agrupamiento social, como construcciones culturales en sus diversos niveles de evolución, mientras que el marxismo, la correlaciona con otros conceptos como hegemonía, determinación, infraestructura y superestructura. Todas estas acepciones convergen en que la cultura es la piedra angular donde se asientan la antropología cultural y la social, y su importancia es innegable para conocer, comprender y valorar las actitudes de cualquier individuo.

Referirse a cultura es hablar de que en el mundo actual todo es cultural, y que no surge por obligación, sino por las necesidades de los seres humanos. Cada sociedad comparte por sí misma, y no por condicionantes, el mismo lenguaje, las creencias religiosas, las tradiciones, la arquitectura, la ciencia, el arte, la literatura, entre otras, y eso es cultura.

Néstor García Canclini define la cultura como el conjunto de procesos donde se elabora la significación de las estructuras sociales, se la reproduce y transforma mediante operaciones simbólicas. Estas aproximaciones al concepto de cultura se traducen en lo que Canclini llama la teoría de la ideología, donde afirma que ambas teorías se necesitan, para correlacionar los procesos culturales, con sus condiciones sociales y producciones significantes (Canclini, 2012). Toda producción significante es susceptible de ser analizada y explicada. La ideología, por tanto, no es ni condicionante, ni determinada, es más bien una consecuencia de la misma cultura.

En estas aproximaciones teóricas, referirse a Bourdieu es hablar del sentido sociológico de la cultura; Pierre Bourdieu (2010) es uno de los autores más interesantes de la posguerra, que plantea la relación entre los valores y prácticas adquiridos y compartidos por un grupo de personas, con un fin específico. Esta definición omite aquella antropológica que explica la relación de naturaleza/cultura, y engloba diferentes acepciones, desde los correspondientes a los llamados bienes culturales, como las prácticas sociales de colectivos o grupos sociales que comparten rasgos culturales en común. Bourdieu, aunque no se acepte totalmente, llama particularmente la atención por la lucha simbólica entre las clases y por la imposición de sus valores culturales. Hablar entonces de las relaciones entre lo antropológico y lo sociológico es referirse a la problemática teórica sobre las definiciones de la cultura y su inserción en el pensamiento contemporáneo.

Estas definiciones parten de hechos sociológicos y se conectan, de modo casi natural, con fenómenos que se dan por la convergencia de los procesos políticos, económicos y sociales; uno de estos fenómenos se desarrolla en la segunda mitad del siglo XX, gracias a la interdependencia de las naciones y a las nuevas conexiones más allá de lo económico; este fenómeno se conoce como globalización que, aunque estuvo alejada de lo cultural, solo por definición encontró en lo cultural una alternativa lucrativa y determinante, en una combinación nociva con lo político y lo económico.

\section{LA CULTURA Y LA GLOBALIZACIÓN}

Desde un enfoque sociocultural, la globalización está ligada a la generalización de valores culturales; es un aspecto de un fenómeno más amplio que afecta a todas 
las dimensiones de la condición humana. Con la globalización se da inicio a las nuevas configuraciones de región en los ámbitos político, económico, social y cultural, que establecieron nuevos procesos estructurales en la mayoría de los países, y a la conformación de bloques económicos, como plataformas de desarrollo.

La globalización es un hecho concreto que está cambiando por completo las estrategias económicas de todas las naciones, redefiniendo las relaciones internacionales y creando nuevos y poderosos patrones culturales; patrones que de manera asimétrica favorecen a ciertos grupos de interés y ciertos valores (Krebs \& Schmidt, 1999).

Si se entiende el término globalización, y se examinan las estructuras políticas de los países privilegiados, se encontrarán procesos en los que cada uno, a través de sus gobiernos, defiende sus intereses de una manera firme y contundente. Pero si se mira desde la perspectiva de los que no son tan beneficiados, se encuentra una cadena de sucesos, que en la búsqueda por la protección de los bienes culturales, han modificado sus estructuras sin objetivos concretos y bajo la hegemonía de patrones culturales dominantes. La segunda mitad del siglo XX mostró que frecuentemente las políticas homogeneizadoras son improductivas y generan ingobernabilidad.

La globalización incide de alguna manera en la sociedad y, de hecho, en la cultura. Cuando se analiza la economía dentro del capitalismo global, se encuentran elementos como la productividad, la innovación y la creatividad, y al mismo tiempo surge un elemento que es la redefinición del trabajo (Villaroel, 2001). La globalización ha flexibilizado a las organizaciones; según Villarroel, el fácil acceso a las redes ha permitido que se creen nuevos mercados, pero al mismo tiempo se han generado nuevos valores culturales, que han inducido a la homogeneización.

\section{LAS INDUSTRIAS CULTURALES Y EL EMPRENDIMIENTO CULTURAL}

Las industrias culturales son una respuesta a la homogeneización y constituyen un componente cada vez más importante en las economías basadas en el conocimiento. No solo contribuyen al crecimiento económico, sino que también actúan como elementos vehiculares en la transmisión de la identidad cultural. Durante los diez últimos años, se ha empezado a reconocer este hecho y se han desarrollado políticas específicas para la promoción de las industrias culturales. Este creciente interés por este tipo de industrias ha supuesto la proliferación de análisis entre las industrias culturales y el desarrollo económico de las naciones. La Unesco, a principios del 2008, publicó el primer informe sobre economía creativa, donde se discuten las oportunidades de desarrollo que venían con la cultura y la creatividad. Hablaba, asimismo, de la economía creativa, como un importante posibilitador de la generación de empleo, del desarrollo de la innovación y de la inclusión social, como una consecuencia de las construcciones sociales (Unesco, 2010).

Las industrias culturales agregan valor económico y social a las naciones e individuos, y constituyen una forma de conocimiento que se traduce en empleos y grandes oportunidades de desarrollo. 
En el documento Conpes (2010), que contiene la política para la promoción de las industrias culturales en Colombia, se desarrolla un análisis de la situación de las industrias culturales, y se presentan cinco ejes problemáticos como la circulación y distribución de bienes y servicios, el bajo acceso a la información, el escaso desarrollo de las industrias culturales y el incipiente uso de las nuevas tecnologías en el desarrollo de los modelos de negocio. Estos aspectos, combinados con la falta de interés de ciertos sectores de la economía por invertir en la cultura, se convierten en una serie de elementos que conforman la cortina gris de las industrias culturales.

Max Horkheimer y Theodor Adorno hablaron del término de industria cultural, como una crítica al entretenimiento de masas, hecha por la Escuela de Frankfurt. Los autores afirman que aquellos que tienen intereses en la industria cultural se valen de términos tecnológicos. Esto equivale a que se imponen métodos de reproducción que conducen inevitablemente a la falta de generar productos únicos (Horkheimer $\mathcal{E}$ Theodor, 1988). Para la Unesco, las industrias culturales son aquellas que combinan la creación, y comercialización de contenidos abstractos, pero de índole cultural. Estos productos están centrados en promover la diversidad cultural y asegurar el acceso democrático. (Unesco, 2010).

\section{LAS MEDIATECAS Y EL EMPRENDIMIENTO CULTURAL}

Según el Consejo Internacional de Museos, creado en 1946, como una red única de más de 30000 museos alrededor del mundo, el tráfico ilícito de bienes culturales ocupa el tercer puesto de las actividades criminales en el mundo. No es entonces, extraño entender cómo diferentes organizaciones alrededor del mundo trabajan constantemente por salvaguardar el patrimonio cultural de las naciones.

El museo, según el Consejo Internacional de Museos, tiene como función preservar y generar valor a las colecciones que constituyen un importante patrimonio público (ICOM, 2013). Es un espacio dotado de identidad que inscribe dentro de sus objetivos, hacer propios los valores culturales, que deben ser trasmitidos de generación en generación, y que a través de la puesta en escena confluyen en una dinámica que posibilita el intercambio cultural.

En Colombia, por ejemplo, el interés por salvaguardar y exhibir el patrimonio viene del siglo XIX. El 28 de julio de 1823, el Congreso de la República expidió la Ley de Creación del Museo Nacional, ley con la que se ordena la creación de una escuela y un museo de mineralogía (Ministerio de Cultura, 2010).

Los museos tienen obligaciones especiales relacionadas con la protección, accesibilidad e interpretación de los testimonios esenciales de la cultura. De acuerdo con los

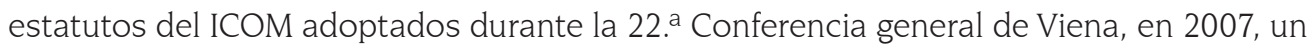
museo es una institución permanente, sin fines de lucro y abierta al público, que adquiere, conserva, expone y difunde el patrimonio material e inmaterial de la humanidad con fines de estudio, educación y recreo. El museo se convierte en la mayor manifestación de identidad cultural de un territorio. 
En los últimos años, el uso de las nuevas tecnologías en museos, galerías y salas de exhibición, ha aumentado considerablemente. Según el ICOM, las visitas virtuales superan las visitas físicas, y se visualizan como una interesante alternativa, que combina diferentes elementos que mejoran la experiencia del visitante. Conferencias, seminarios, foros, debates, tiendas online, visitas al museo, entre otros, completan una oferta que hace de la dinámica del museo una experiencia que supera el formato. Pero cuando el museo se convierte en un espacio dinámico, soporte de formatos audiovisuales, explora lenguajes que permiten una interacción directa entre el creador y el usuario, almacena información que puede ser consultada en cualquier momento y desde cualquier lugar se le denomina mediateca.

Las mediatecas, así como los museos, requieren de una planificación estratégica, a través de la cual se establecen los lineamientos para funcionar. Deben tener identificado su público objetivo y diseñar estrategias para captar la atención de los usuarios. Dentro de los elementos más importantes para definir el éxito de una mediateca, está la conceptualización y posicionamiento de su imagen, como recurso invaluable de comunicación (Museo Nacional de Colombia, 2013).

\section{MEDIATECA COMO PROPUESTA DE MODELO DE EMPRENDIMIENTO PARA CREADORES EMERGENTES}

La economía del conocimiento da paso a nuevas formas de producción de bienes tangibles e intangibles. El capital intelectual se constituye en una inagotable fuente de producción que, combinado con las tecnologías de la información y la comunicación, se convierten en una interesante estrategia para aquellos que se encuentran inmersos en la cultura (Unesco, 2010).

Las comunicaciones inmediatas han logrado cerrar la grieta que separa a muchos artistas de la visibilidad. El rol de los museos, y en este caso de una mediateca, es actuar como preservadores de objetos (artísticos y culturales), pero también divulgadores ante la constante pérdida de la tradición cultural. Año tras año, en Colombia, por ejemplo, surgen artistas que nunca llegan a ser reconocidos. Estrategias como el salón de artistas del Ministerio de Cultura buscan generar espacios para que los artistas tengan el reconocimiento que merecen. Asimismo, el Ministerio de Cultura, a través de la política de emprendimiento cultural, se centra en la generación de un entorno adecuado para la creación y el fortalecimiento de proyectos culturales sostenibles, como factor relevante para el desarrollo local.

La mediateca, desde la investigación realizada en la Unipanamericana, (Fundación Universitaria Panamericana), propone un modelo de espacio virtual en el que se almacenen y publiquen los trabajos como resultado de ejercicios académicos, en la Facultad de Comunicación. El proyecto parte de una primera fase, con el estado del arte de las mediatecas en el mundo y que de una u otra manera tienen relación con el área disciplinar de la comunicación. Luego se desarrolla un proceso de identificación, donde se plantea 
una segunda fase de categorización, que responda a las necesidades de la Institución y específicamente de la Facultad de Comunicación. Esta categorización parte de encuestas aplicadas a los estudiantes de la Institución, entrevistas a expertos en diseño y desarrollo de mediatecas, y profesionales relacionados con la catalogación, categorización y valoración de la información (Bonilla et al., 2012).

Según el Manifiesto de la Federación Internacional de Asociaciones de Bibliotecarios y Bibliotecas (IFLA) sobre las bibliotecas digitales, es importante que se desarrollen estrategias que permitan subsanar la brecha digital y acceder a los recursos informativos y tecnológicos, para contribuir al desarrollo de las naciones. En este sentido las instituciones académicas deben proporcionar parte de su capital intelectual, y contribuir a la democratización de la cultura (Unesco, 2011).

Dentro de la metodología empleada en el desarrollo del proyecto, se realiza un cruce de variables sobre el contenido programático de los programas que hacen parte de la Facultad de Comunicación de la Fundación Universitaria Unipanamericana: Comunicación Social y Periodismo, Diseño Visual y Mercadeo y Publicidad. Es importante resaltar que la relación existente entre los cuatro programas académicos da cabida al planteamiento que se propone para el ejercicio de documentar, catalogar y posteriormente clasificar la información de una forma clara y que responda a las necesidades de cada uno de los programas.

La categorización, entonces, se establece según el cruce de variables en cuatro grandes categorías: visual, audio, audiovisual y multimedia, y textual. Estas cuatro categorías logran resumir los intereses que cada programa tiene a la hora de almacenar y divulgar su información. Con las respuestas generadas desde las encuestas, se obtiene otra serie de variables que vinculan al estudiante como actor importante del proceso y contribuyen con su producción a la construcción de la memoria cultural en torno a la comunicación.

La mediateca es un instrumento interesante que dota de identidad a la institución, y proporciona los elementos necesarios para contribuir a la construcción de la memoria en torno a la comunicación. Muchos de los trabajos realizados por los estudiantes cumplen un objetivo específico y entran a formar parte de los grandes proyectos que quedan en el olvido. Tener una memoria como parte de un proceso académico será el soporte que evidencie la evolución misma del estudiante, los programas, la Institución y las formas como se han abordado las grandes problemáticas de la comunicación. Entonces son los mismos estudiantes quienes han reconocido, al estar directamente involucrados en el desarrollo del proyecto de la mediateca, que deben estar claras las reglas de la apropiación del conocimiento cuando una obra hace parte de repositorio digital.

La iniciativa del año 2010, de las bibliotecas digitales europeas, se relaciona con la iniciativa de la mediateca; cuando en 2005 fue lanzada por la Dirección General de la Sociedad de Información y Media de la Comisión Europea, posibilitó la conversión de miles de formatos físicos a formatos digitales. Este proyecto facilitó la apropiación social y cultural del conocimiento, y lo dejó a disposición del público. (Unesco \& Cerlalc, 2008). 
¿Pero que sucede cuando el almacenamiento y la memoria no son suficientes, y los trabajos de los estudiantes terminan haciendo parte de una construcción estática del conocimiento? La mediateca, desde la comunicación como modelo de emprendimiento, busca que no solo sean los estudiantes quienes publiquen en la plataforma virtual. Se invita a participar a docentes, artistas, productores, diseñadores, mercadólogos, comunicadores, publicistas, entre otros, que no han tenido la posibilidad de exponer sus obras, y que puedan publicar en una mediateca para creadores emergentes (Bonilla et al.).

Entablar una conexión directa con el creador de la obra, conocer la retrospectiva y prospectiva, sobre la forma de comunicamos, dinamizar la gestión cultural y apropiarse del bien cultural, contribuir a la protección del patrimonio cultural son solo pequeñas acciones que se derivan de la creación e implementación de la mediateca como modelo de emprendimiento cultural, pues el reconocimiento de artista confluye en una dinámica donde de una manera segura puede comercializar el bien cultural.

La investigación en comunicación es también parte importante de la mediateca. Estados de arte que terminan en los anaqueles de la bibliotecas, proyectos de investigación que no llegan a ser reconocidos y alianzas estratégicas de trabajo colaborativo que nunca llegan a término son otras tantas acciones importantes como resultado del desarrollo de la mediateca.

¿Y qué papel juega el consumidor de la mediateca? ¿Cómo se comporta el consumidor del bien cultural? Aunque por décadas, su papel ha sido previsible, y según Mattelart, al momento de consumir todos los hombres son iguales, con el bien cultural no siempre sucede lo mismo. El consumidor de bienes simbólicos tiene un rol interpretativo y autónomo, cuyo componente más importante es su comportamiento (Torres, 2005).

Según las políticas para la creatividad de la Unesco, el emprendimiento cultural debe darse desde seis frentes: el primero es la competitividad y la innovación; el segundo, el recurso humano; el tercero, la infraestructura y las inversiones; el cuarto, la financiación; el quinto, el marco normativo, y el sexto, el desarrollo de mercados. Estos frentes deben estar acompañados de la coordinación de esfuerzos y de una eficaz gestión para la continuidad (Unesco, 2010). Desde esta perspectiva una mediateca como modelo de emprendimiento cultural debe responder de manera clara a cada uno de estos frentes para dar satisfacción a las necesidades de posicionamiento y comercialización de los actores interesados. La competitividad y la innovación, por ejemplo, se dan desde la forma en que se cataloga y clasifica el bien cultural para que sea apropiado, y desde la manera como se puede contactar al creador de la obra y diversificar el bien cultural más allá del formato.

En 2005, la Conferencia General de la UNESCO reconoce la Convención sobre la Protección y la Promoción de la Diversidad de las Expresiones Culturales. Es la primera vez que un tratado internacional se encarga de analizar los efectos de la globalización sobre la protección de los bienes culturales y promueve el fortalecimiento de las industrias culturales (Unesco, 2010). 
Según la Unesco, la globalización y la revolución digital han dado un papel importante al sector cultural. Estas industrias, cuyo mayor capital es el bien simbólico, se están convirtiendo en un sector estratégico para el desarrollo productivo, la competitividad y el empleo. Según este informe, se estima que las industrias culturales contribuyen con cerca del 3,4\% del PIB mundial.

Es una tarea importante para países como Colombia fortalecer sus sectores culturales a la luz de los informes de la Unesco. Herramientas como una mediateca, y en este caso específico de la comunicación, se perfilan como modelos de emprendimiento cultural, que haciendo uso de las nuevas tecnologías, facilitan el acceso a la democratización de la cultura.

Las instituciones académicas deben fortalecer sus procesos de investigación y producción, y no olvidar que dentro de la generación de conocimiento, el capital social y cultural determina de alguna manera los rasgos materiales, intelectuales y emocionales que identifican a las naciones (Unesco, 2010).

\section{CONCLUSIONES}

La Unesco es la única organización que reconoce e insiste en que la cultura debe ser un campo prioritario de investigación en los países y, aunque dispone de políticas claras en relación con las industrias culturales, no tiene en la actualidad una metodología que permita medir la participación de las industrias culturales.

La prioridad para los actores involucrados en el emprendimiento cultural, debe ser la generación de una masa crítica de experiencias, conocimientos y datos que demuestren de manera irrefutable la contribución de la cultura al desarrollo económico de las naciones. Los países en vía de desarrollo deberán convertir las dinámicas de la globalización en herramientas participativas, que potencien la inyección de los valores de cada nación en lo global. Para esto es importante tener unas políticas claras que protejan el patrimonio cultural de cada territorio.

Una mediateca como modelo de emprendimiento cultural para creadores emergentes descentraliza la economía de lo cultural, y la focaliza en una estrategia que posibilite la democratización de la cultura. El lenguaje de lo tecnológico frente a lo cultural convergen en el desarrollo de nuevos lenguajes que hacen de las obras productos dinámicos que trasmiten identidad, más allá del formato.

Vincular dimensiones abstractas, derivadas de la cultura, con dimensiones más concretas relacionadas con la economía se visualiza como motores de innovación y como ventajas competitivas en un mercado globalizado. Diseñar una mediateca permitirá que los actores relacionados con la cultura potencien el emprendimiento cultural en un espacio seguro, vigilante, y propio de las áreas relacionadas con la comunicación; conectarse de manera directa con los creadores de las obras permitirá al mismo tiempo potenciar la generación de conocimiento y la construcción en un espacio colaborativo, capaz de contribuir a la protección del patrimonio cultural. 
Analizar la globalización desde la cultura es realmente importante, cuando lo que está en juego es la protección del patrimonio cultural de las naciones. Es necesario concebir la cultura como una dimensión innegable en la construcción del conocimiento.

\section{REFERENCIAS BIBLIOGRÁFICAS}

Álvarez y Urbano. (2013). Diversidad Cultural y emprendimiento. Revista de Ciencias Sociales.

Bonilla, J., Cabrera, C., Crespo, N., Cristiano, H., Rios, B., E Sanchez, W.(2012). Propuesta de categorización para la Mediateca de Comunicación de Unipanamericana (Tesis de pregrado). Unipanamericana Fundación Universitaria, Bogotá Colombia.

Bourdieu, P. (2010). El sentido social del gusto: elementos para una sociología de la cultura. Buenos Aires: Siglo XXI.

Canclini, N. G. (2012). Ideología, cultura y poder. Buenos aires: Secretaría de extensión universitaria.

Castells, M. (2005). La globalizacion. Santiago de Chile: Chile Tecnológico.

Conpes. (2010). Documento conpes 3659. Bogotá: Conpes.

Horkheimer, M., \& Theodor, A. (1988). Dialéctica del iluminismo. Buenos Aires: Sudamericana.

ICOM. (2013). Código de deontología del ICOM para los museos. Barcelona: ICOM.

Krebs, M., \& Schmidt-Hebbel, K. (1999). Patrimonio cultural: aspectos económicos y políticas de protección. Perspectivas de política, economía y gestión, (2), 207-245.

Mattelart, A. (1998). La mundialización de la comunicación. Barcelona: Páidos Ibérica.

Ministerio de Cultura. (2010). Conpemdio de políticas culturales. Bogotá: Ministerio de Cultura.

Museo Nacional de Colombia. (2013). Manual de gestión y competitividad para los museos colombianos. Bogotá: Museo Nacional de Colombia.

Spradley, J. y. (1975). Antropology: The cultural perspective. New York: Wiley.

Torres, M. L. (2005). Reflexión sobre el consumidor de las industrias culturales. Universidad y empresa, 7 .

Unesco \& Cerlalc. (2008). Bibliotecas digitales. Unesco.

Unesco. (2001). Declaración universal sobre la diversidad cultural. Johannesburgo: Serie sobre la Diversidad Cultural $\mathrm{N}^{\circ} 1$.

Unesco. (2010). Economía creativa. Concepción, Chile: Unesco.

Unesco. (2010). Políticas para la creatividad. Argentina: Unesco.

Unesco. (2011). Manifiesto de la federación internacional de asociaciones de bibliotecarios y bibliotecas (IFLA) sobre las bibliotecas digitales. Conferencia general (pág. 6). Paris: Unesco.

Villaroel, M. E. (2001). Globalización, Cultura y Exclusión social. Venezuela: Fermentun.

Williams, R. (1980). Marxismo y literatura. Barcelona: Peninsula. 